\title{
Atherogenic index of plasma (AIP): a novel predictive indicator for the coronary artery disease in postmenopausal women
}

Ting-Ting $\mathrm{Wu}^{1+}$, Ying $\mathrm{Gao}^{2 \dagger}$, Ying-Ying Zheng ${ }^{3}$, Yi-Tong $\mathrm{Ma}^{1 *}$ and Xiang Xie ${ }^{1 *}$ (D)

\begin{abstract}
Background: Dyslipidemia is one of the most important factors for coronary artery disease (CAD). Atherogenic index of plasma (AIP) is a novel indicator involved in dyslipidemia. However, the relation between AIP and CAD in postmenopausal women remains unclear. We hypotheses that AIP is a strong predictive indicator of CAD in postmenopausal women.

Methods: A propensity score matching case-control study including 348 postmenopausal CAD cases and 348 controls was conducted in the present study.

Results: Compared with controls, CAD patients had higher levels of total cholesterol (TC), triglyceride (TG), lowdensity lipoprotein cholesterol (LDL-C) and apolipoprotein B (APOB), but lower high-density lipoprotein cholesterol (HDL-C) and apolipoprotein A-1 (APOA-1). The values of nontraditional lipid profiles, including non-HDL-C, TC/HDL-C, LDL-C/HDL-C, non-HDL-C/HDL-C (atherogenic index, Al), TC*TG*LDL/HDL-C (lipoprotein combine index, LCl), $\log (\mathrm{TG} / \mathrm{HDL}-\mathrm{C}$ ) (atherogenic index of plasma, AIP) and APOB/APOA-1 were all significantly higher in the CAD patients. The results of Pearson correlation analyses showed AIP was positively and significantly correlated with TC $(r=0.092, P<0.001)$, TG $(r=0.775, P=0.015)$, APOB $(r=0.140, P<0.001)$, non-HDL-C $(r=0.295, P<0.001)$, TC/HDL-C $(r=0.626, P<0.001)$, LDL-C/HDL-C $(r=0.469, P<0.001), \mathrm{Al}(r=0.626, P<0.001), \mathrm{LCl}(r=0.665, P<0.001)$, APOB/APOA-1 $(r=0.290, P<0.001)$ and was negatively correlated with APOA-1 $(r=-0.278, P<0.001)$ and HDL-C $(r=-0.665, P<0.001)$. In the multivariate logistic regression analysis, AIP was an independent predictor of CAD. After adjusting for the traditional clinical prognostic factors including diabetes and hypertension, we found AIP could be an independent risk factor for CAD (odds ratio [OR], 3.290; 95\% confidence interval [CI], 1.842-5.877, $P<0.001$ ). After adjusting for multiple clinical factors include diabetes, hypertension, smoking, heart ratio, fasting blood glucose, we found AIP also could a powerful risk factor, OR=3.619, 95\%Cl (2.003-6.538), $P<0.001$.
\end{abstract}

Conclusion: The present study indicated that AIP might be a strong marker for predicting the risk of CAD in postmenopausal women.

Keywords: Atherogenic index of plasma, Coronary artery disease, Postmenopausal women, Lipids

\footnotetext{
*Correspondence: myt_xi@sina.com; xiangxie999@sina.com

${ }^{\dagger}$ Ting-Ting Wu and Ying Gao contributed equally to this work.

${ }^{1}$ Department of Cardiology, First Clinical Medical College \& First Affiliated

Hospital of Xinjiang Medical University, Urumqi 830054, People's Republic of

China

Full list of author information is available at the end of the article
}

(c) The Author(s). 2018 Open Access This article is distributed under the terms of the Creative Commons Attribution 4.0 International License (http://creativecommons.org/licenses/by/4.0/), which permits unrestricted use, distribution, and reproduction in any medium, provided you give appropriate credit to the original author(s) and the source, provide a link to the Creative Commons license, and indicate if changes were made. The Creative Commons Public Domain Dedication waiver (http://creativecommons.org/publicdomain/zero/1.0/) applies to the data made available in this article, unless otherwise stated. 


\section{Background}

The ability of plasma lipid to migrate into the subintimal layer is an important step to developing atherosclerosis. Lipid and its lipoprotein constituent have been designated as a mediator and a marker of coronary heart disease (CAD). It is characterized by high ratio of low-density lipoprotein cholesterol (LDL-C) to high-density lipoprotein cholesterol (HDL-C) and increased level of triglycerides (TG) [1]. The association between lipoprotein concentration and cardiovascular disease (CVD) risk has been studied by numerous authors for decades, and total cholesterol (TC) and LDL-C are well known cardiovascular risk factors. Elevated TG and a low HDL level are strong markers of cardiovascular diseases. An increase of TG levels causes an increase in the small dense LDL (sdLDL) level and finally causes an increase of cardiovascular risk [2-5]. Recently, it was determined that the atherogenic index of plasma (AIP) value, which is acquired by the logarithmic transformation of the number found by dividing plasma TG value to HDL value, can be a good marker for the risk of atherosclerosis and cardiovascular disease [6-8]. It was reported that an $\mathrm{AI} P$ value of $0.1-$ 0.24 shows medium cardiac risk [9].

Menopause, which is the permanent cessation of menstruation following loss of ovarian activity, has considerable impact on social, reproductive, physical and psychological health of the woman. It has been shown that postmenopausal women have less cardiovascular friendly lipid profiles than before menopause [10]. Data from cross-sectional and longitudinal studies have shown that menopause alters CVD risk factors. Postmenopausal compared with premenopausal women have higher plasma TC, LDL-C, very low-density lipoprotein cholesterol (VLDL-C), and TG levels [11].There is convincing evidence that menopause is associated with central adiposity [12], increased diastolic blood pressure [13] and increased insulin resistance [14], hence an increased likelihood to develop CVD. Thus, the true value of the AIP is yet to be determined in postmenopausal women. To this end, we undertook the present study, which aimed at investigating the link between AIP and risk of CAD and finding out whether AIP might be a better marker for predicting the risk of CAD among postmenopausal women.

\section{Methods}

\section{Study population}

The exclusion criteria were the following: patients who had undergone a bilateral oophorectomy or a hysterectomy, with or without ovarian preservation; patients who did not undergo a coronary artery angiography (CAG) examination; incomplete data for traditional lipid profiles; and patients with hypothyroidism or nephrotic syndrome. According to the exclusion criteria, 1006 consecutive premenopausal women with 600 CAD patients, 406 healthy controls were enrolled in this study between January 2012 and January 2015 who were hospitalized in the First Affiliated Hospital of Xinjiang Medical University. After a propensity score matching for age, a total of 348 CAD cases and 348 apparently healthy premenopausal women served as control subjects were enrolled in this study. The study protocol was approved by the Ethics Committee of the First Affiliated Hospital of Xinjiang Medical University. A flowchart outlining our study was shown in Fig. 1.

\section{Definition}

Essential hypertension(EH) was defined as a systolic blood pressure of $\geq 140 \mathrm{mmHg}$ and/or a diastolic blood pressure of $\geq 90 \mathrm{mmHg}$ in at least 2 measurements or use of any antihypertensive drug. Diabetes mellitus (DM) was defined as fasting plasma glucose levels $126 \mathrm{mg} / \mathrm{dl}$ on multiple measurements or current use of anti-diabetic medications. Smoking status was defined as current tobacco use. Menopause was defined as amenorrhoea for at least 12 months and elevated gonadotrophin levels $(\mathrm{FSH}>40 \mathrm{IU} / \mathrm{l})$.CAD was defined in accordance with the 1979 WHO diagnostic criteria [15], and all of the patients underwent a CAG examination. The CAG examinations were performed using Judkin technique via the radial or femoral artery. Angiograms were analyzed by 2 experienced doctors who were blinded to this study. Control subjects were defined as those lacking typical angina pectoris symptoms and those in whom stenosis of the major coronary arteries was less than $50 \%$.Scoring of CAD severity was performed with a modification of the coronary atherosclerosis scoring system named the standard of Gensini method, as described by Gensini GG [16]. The Gensini score is an anatomic scoring system and quantifies the properties of lesions including their complexity, morphology, and locations in the coronary tree. Gensini score was used to calculate the levels of coronary severity scores (CSS).

The AIP is defined as the base 10 logarithm of the ratio of the concentration of TG to HDL-C, where each concentration is expressed in $\mathrm{mmol} / \mathrm{L}$; The non-HDL-C is defined as TC minus HDL-C; The atherogenic index (AI) is defined as the ratio of non-HDL-C to HDL-C; The lipoprotein combine index $(\mathrm{LCI})$ is defined as the ratio of TC*TG*LDL to HDL-C.

\section{Laboratory assessments}

Peripheral venous blood samples of the patients were obtained on admission in the inpatient ward. Data regarding clinical and demographic characteristics including age, history of hypertension and diabetes mellitus and smoking status were collected from medical records. Laboratory data including urea nitrogen, creatinine, uric 


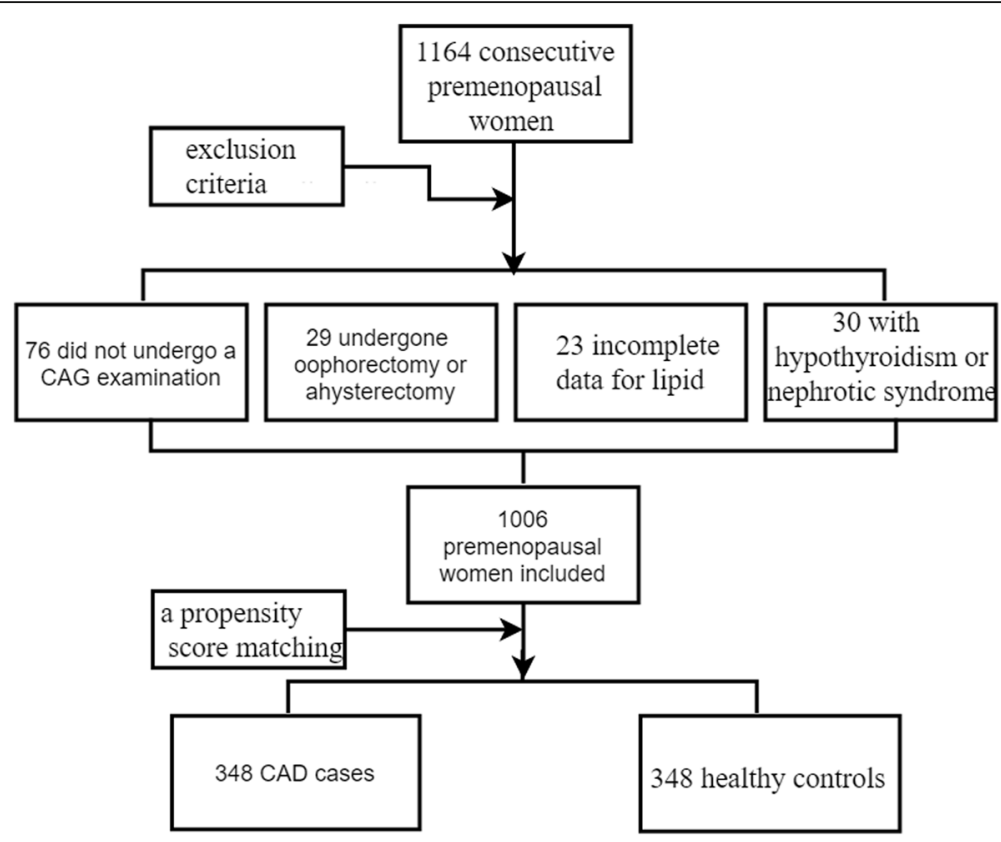

Fig. 1 The flow chart of the study

acid, fasting blood glucose, bilirubin and lipid parameters as TC, TG, HDL-C, LDL-C, APOA-1, APOB were noted.

\section{Statistical analysis}

Statistical analyses were carried out in SPSS 22.0 Statistical Package Program for Windows (SPSS Inc., Chicago, Illinois). The non-HDL-C, TC/HDL-C, LDL-C/HDL-C, $\mathrm{LCI}, \mathrm{AI}, \mathrm{AIP}$ and APOB/APOA-1concentration ratios were calculated. Continuous variables were presented as the mean \pm standard deviation (SD) and were compared using an independent samples $t$ test. However, if the data were not accorded with normal distribution, nonparametric test should be used. Categorical variables were expressed as frequencies and percentages and were compared using Chi-square tests. The correlation between the AIP values and other variables were calculated by Pearson correlation analysis. Both univariate and multivariate logistic regression analyses were performed to explore the relationship between the lipid parameters and risk of CAD. The adjusted odds ratio (OR) per $1 \mathrm{SD}$ increase in the corresponding lipid variable and 95\% confidence intervals $(95 \% \mathrm{CIs})$ were calculated. A value of $P<0.05$ in a 2 -sided test was considered significant.

\section{Results}

\section{Basic characteristics and cardiovascular risk factors of} subjects

After a propensity score matching for age, a case-control study includes a total of 696 postmenopausal women were enrolled in this study, and the mean age of subjects is $61.7 \pm 7.1$. We can see that subjects diagnosed with CAD were more likely to have higher known history of hypertension, diabetes mellitus and smoking. Higher heart rate, fasting blood glucose and higher traditional lipid parameters as TG, TC, LDL-C, APOB, and lower HDL-C, APOA-1.The median of LCI was16.27, and after a normal test being carried out, a nonparametric test was used in LCI $(P<0.001)$. The nontraditional lipid profiles were much significantly higher in $C A D$ cases $(P<0.05)$. The distribution of various clinical and biological variables measured is depicted by Table 1 .

\section{Correlation between AIP and other parameters}

Pearson correlation analyses were performed to investigate the correlation of AIP with other continuous variables. As summarized in Table 2, AIP was positively and significantly correlated with TC $(r=0.092, P<0.001)$, TG $(r=0.775, \quad P=0.015), \quad$ APOB $\quad(r=0.140, \quad P<0.001)$, non-HDL-C $(r=0.295, P<0.001)$, TC/HDL-C $(r=0.626$, $P<0.001), \quad$ LDL-C/HDL-C $(r=0.469, P<0.001)$ AI $(r=0.626, \quad P<0.001), \mathrm{LCI} \quad(r=0.665, \quad P<0.001), \quad \mathrm{APOB} /$ APOA-1 $(r=0.290, \quad P<0.001)$ and was negatively correlated with APOA-1 $(r=-0.278, P<0.001)$ and HDL-C $(r=-0.665, P<0.001)$.

AIP was also positively and significantly correlated with $\mathrm{EH}(r=0.087 ; p=0.021)$, DM $(r=0.164 ; p<0.001)$, Gensini $\operatorname{score}(r=0.133 ; p<0.001)$, but not with age $(r=-0.057 ; p=0.135)$, active smoking $(r=-0.013$; $p=0.742)$, BMI $(r=0.039 ; p=0.304)$, SBP $(r=0.053 ; p=$ $0.166), \mathrm{HR} \quad(r=-0.035 ; p=0.363), \mathrm{BUN}(r=-0.003 ; p=$ $0.947)$, uric acid $(r=-0.010 ; p=0.785)$, FBG $(r=-0.039$; 
Table 1 Baseline characteristics of the both CAD and control group

\begin{tabular}{|c|c|c|c|c|}
\hline Variables & $\operatorname{CAD} \operatorname{cases}(n=348)$ & Controls $(n=348)$ & $t\left(x^{2}\right)$ & $P$ value \\
\hline$\overline{E H}[n(\%)]$ & $213(61.2 \%)$ & $177(50.9 \%)$ & 7.558 & 0.006 \\
\hline DM [n(\%)] & $89(25.6 \%)$ & $62(17.8 \%)$ & 6.165 & 0.013 \\
\hline Smoking [n(\%)] & $67(19.5 \%)$ & $43(12.4 \%)$ & 6.478 & 0.011 \\
\hline Heart Rate (bpm) & $76 \pm 11$ & $74 \pm 11$ & 0.173 & 0.031 \\
\hline $\mathrm{SBP}(\mathrm{mmHg})$ & $130 \pm 15$ & $129 \pm 9$ & 37.274 & 0.232 \\
\hline BUN (mmol/L) & $5.47 \pm 1.59$ & $5.44 \pm 1.57$ & 0.039 & 0.824 \\
\hline Creatinine (mmol/L) & $74.06 \pm 19.03$ & $72.68 \pm 15.60$ & 4.015 & 0.296 \\
\hline Uric acid (mmol/L) & $324.46 \pm 85.22$ & $315.24 \pm 95.94$ & 1.433 & 0.181 \\
\hline FBG (mmol/L) & $6.32 \pm 2.76$ & $5.85 \pm 2.21$ & 8.138 & 0.013 \\
\hline TBIL (mmol/L) & $12.83 \pm 7.08$ & $12.28 \pm 6.28$ & 4.421 & 0.283 \\
\hline DBIL (mmol/L) & $4.22 \pm 3.64$ & $3.73 \pm 3.34$ & 1.980 & 0.066 \\
\hline IBIL (mmol/L) & $8.67 \pm 6.30$ & $8.57 \pm 5.13$ & 6.887 & 0.822 \\
\hline BMI $\left(\mathrm{kg} / \mathrm{m}^{2}\right)$ & $26.27 \pm 3.29$ & $25.84 \pm 3.83$ & 6.201 & 0.111 \\
\hline \multicolumn{5}{|c|}{ Traditional lipid profiles } \\
\hline $\mathrm{TG}(\mathrm{mmol} / \mathrm{l})$ & $1.89 \pm 1.34$ & $1.65 \pm 0.93$ & 2.239 & 0.007 \\
\hline $\mathrm{TC}(\mathrm{mmol} / \mathrm{l})$ & $4.56 \pm 1.20$ & $4.39 \pm 0.98$ & 9.530 & 0.043 \\
\hline $\mathrm{HDL}-\mathrm{C}(\mathrm{mmol} / \mathrm{l})$ & $1.09 \pm 0.29$ & $1.20 \pm 0.32$ & 0.812 & $<0.0001$ \\
\hline LDL-C(mmol/l) & $2.82 \pm 1.00$ & $2.61 \pm 0.85$ & 10.801 & 0.003 \\
\hline APOA-1 (g/l) & $1.27 \pm 0.25$ & $1.31 \pm 0.24$ & 0.619 & 0.048 \\
\hline $\mathrm{APOB}(\mathrm{g} / \mathrm{l})$ & $0.93 \pm 0.31$ & $0.87 \pm 0.25$ & 15.384 & 0.009 \\
\hline \multicolumn{5}{|c|}{ Nontraditional lipid profiles } \\
\hline non-HDL-C & $3.47 \pm 1.28$ & $3.20 \pm 0.94$ & 11.971 & 0.001 \\
\hline $\mathrm{TC} / \mathrm{HDL}-\mathrm{C}$ & $4.43 \pm 1.56$ & $3.95 \pm 1.79$ & 2.136 & $<0.0001$ \\
\hline LDL-C/HDL-C & $2.72 \pm 1.12$ & $2.35 \pm 1.16$ & 3.040 & $<0.0001$ \\
\hline $\mathrm{Al}$ & $3.43 \pm 1.56$ & $2.95 \pm 1.79$ & 2.136 & $<0.0001$ \\
\hline AIP & $0.20 \pm 0.27$ & $0.10 \pm 0.27$ & 0.434 & $<0.0001$ \\
\hline APOB/APOA-1 & $0.75 \pm 0.26$ & $0.68 \pm 0.22$ & 13.345 & $<0.0001$ \\
\hline
\end{tabular}

$A$ l atherosclerosis index, AIP atherogenic index of plasma, $C A D$ coronary artery disease, $D M$ diabetes mellitus, $E H$ essential hypertension, $T G$ triglyceride, $H D L-C$ high-density lipoprotein cholesterol, $L C l$ lipoprotein combined index, $L D L-C$ low-density lipoprotein cholesterol, $T C$ total cholesterol, $B M I$ body mass index, $B U N$ Blood urea nitrogen, $F B G$ fasting blood glucose, TBIL total bilirubin, DBIL direct bilirubin, IBIL indirect bilirubin, $A P O A-1$ apolipoprotein A-1, $A P O B$ apolipoprotein B, $A P O B / A P O A-1$ apolipoprotein $B$ to apolipoprotein A-1 ratio

$p=0.308), \quad \mathrm{CR}(r=0.003 ; p=0.930), \quad \operatorname{TBIL}(r=-0.038$;

$p=0.314), \operatorname{DBIL}(r=-0.058 ; p=0.124)$ nor $\operatorname{IBIL}(\mathrm{r}=-0.010$; $p=0.792)$.

\section{Univariate and multivariate logistic regression analysis}

In univariate logistic regression analysis, we can see all lipid parameters were predictors for CAD risk. To eliminate interference from other confounding factors, multivariate logistic regression analyses were made. In model 1, after adjusting for the traditional clinical prognostic factors including diabetes and hypertension, we found AIP could be an independent risk factor for CAD (odds ratio [OR], 3.290; 95\% confidence interval [CI], 1.842-5.877, $P<0.001$ ). To construct model 2 , univariate models for each of the all predictor variables were run, with $\mathrm{CAD}$ as the outcome variable. Those variables that

Table 2 Correlation between PAI and other variables

\begin{tabular}{|c|c|c|c|c|c|c|c|c|c|c|c|c|c|}
\hline & TC & TG & $\mathrm{HDL}$ & LDL & APOA-1 & APOB & Non-HDL & LDL/HDL & $\mathrm{TC} / \mathrm{HDL}$ & $\mathrm{LCl}$ & $\mathrm{Al}$ & APOB/APOA-1 & Gensini \\
\hline \multirow[t]{3}{*}{ AIP } & $0.092^{*}$ & $0.775^{*}$ & $-0.665^{*}$ & 0.004 & $-0.278^{*}$ & $0.140^{*}$ & $0.295^{*}$ & $0.469^{*}$ & $0.626^{*}$ & $0.665^{*}$ & $0.626^{*}$ & $0.29^{*}$ & $0.133^{*}$ \\
\hline & -0.057 & 0.039 & 0.053 & -0.035 & -0.039 & -0.003 & -0.010 & -0.038 & -0.058 & -0.010 & -0.013 & $0.164^{*}$ & $0.087^{*}$ \\
\hline & Age & BMI & SBP & $H R$ & FBG & BUN & UA & TBIL & DBIL & IBIL & Smoke & DM & $\mathrm{EH}$ \\
\hline
\end{tabular}


were significant $(P<0.05)$ in univariate logistic models were then simultaneously entered into model 2 . After adjusting for multiple clinical factors include diabetes, hypertension, smoking, HR, FBG, we found AIP also could a powerful risk factor $[\mathrm{OR}=3.619,95 \% \mathrm{CI}(2.003-$ $6.538), P<0.001]$ (Table 3).

\section{Discussion}

To our knowledge, there is paucity of literature on its application to postmenopausal women. Therefore, our goal was to assess the association between AIP and CAD and to determine if it might pose an independent risk factor in the postmenopausal women. A case-control study demonstrates that AIP is a usable risk factor in postmenopausal women. Among all lipoprotein markers, AIP was positively associated with Gensini score. AIP independent of smoking habit, history of diabetes mellitus and hypertension was developed as a marker of increased CAD risk that might be better than TC, LDL-C and HDL-C and other lipid concentrations.

We obtained similar AIP values (mean $0.15 \pm 0.27$ ) as Nwagha et al. [17] in their group of Nigerian postmenopausal women (mean $0.15 \pm 0.35$ ), lower than Nansseu et al. [18] Likewise, relation between AIP and known history of diabetes, hypertension and other lipid parameters which have been cited as risks factors for CVD were found $[19,20]$. However, we did not found relation between AIP and BMI, SBP and uric acid, in contradiction with previous reports $[6,7,21]$. Dobiášová et al. [8] demonstrated that AIP inversely correlates with LDL-C particle size in a high diversity population composed of both high and low-risk individuals with high correlation coefficients for the first time. They suggested indeed that
AIP values of -0.3 to 0.1 may be associated with low, 0.1 to 0.24 with medium and above 0.24 with high risk of CVD. It was bolstered that the strong correlation of AIP with lipoprotein particle size may explain its high predictive value of CVD occurrence [9]. AIP was found to be a surrogate for sdLDL particles and negatively associated with LDL-C particle diameter. An increase in AIP indicates a reduction in the LDL particle diameter and a rise in the proportion of sdLDL. SdLDL particles are more prone to oxidation, to promote the formation of foam cells and LDL-C with oxidized apoprotein B is regarded as highly atherogenic characteristic. Also they can cause atherosclerosis by increasing lipid peroxidation, activating oxygen radicals, expressing adhesion molecules on endothelial cells which were linked to endothelial dysfunction [22]. HDL-C allows the use of peripheral cholesterol by transporting it to the liver. Also, it includes antioxidant enzymes such as paraoxonase [23].

This lipoprotein subfraction named AIP is well-known for its strong association with the development of CAD, all-cause mortality, and it also predicts emergency cardiovascular events [6-9, 24-27]. Dobiášová et al. [28] further proved that AIP is strongly associated with atherosclerosis and its complications by establishing an association between the AIP and coronary angiographic findings in patients with CAD. In a prospective study by Onat et al. [19] after a 7.8-year follow-up of 2676 middle-aged adults showed that AIP is a reliable biomarker for predicting CVD morbidity. To support the theory that AIP predicts the risk of development of type 2 diabetes mellitus better than traditional lipid markers, a meta-analysis of 15 studies was done [20].

Table 3 Univariate and multivariate logistic regression analyses for lipid parameters with CAD risk

\begin{tabular}{|c|c|c|c|c|c|c|}
\hline \multirow[t]{2}{*}{ Variables } & \multicolumn{2}{|l|}{ Univariate } & \multicolumn{2}{|l|}{ Model 1} & \multicolumn{2}{|l|}{ Model 2} \\
\hline & OR 95\%Cl & P & OR 95\%Cl & $P$ & OR 95\%Cl & $P$ \\
\hline TG & $1.238(1.056-1.451)$ & 0.008 & $1.197(1.023-1.401)$ & 0.025 & $1.222(1.038-1.438)$ & 0.016 \\
\hline TC & $1.151(1.004-1.320)$ & 0.044 & $1.146(0.998-1.316)$ & 0.053 & $1.147(0.997-1.319)$ & 0.056 \\
\hline $\mathrm{HDL}$ & $0.323(0.195-0.536)$ & $<0.001$ & $0.350(0.210-0.583)$ & $<0.001$ & $0.332(0.198-0.558)$ & $<0.001$ \\
\hline LDL & $1.279(1.086-1.506)$ & 0.003 & $1.278(1.084-1.507)$ & 0.004 & $1.276(1.080-1.508)$ & 0.004 \\
\hline APOA-1 & $0.546(0.299-0.998)$ & 0.049 & $0.558(0.304-1.026)$ & 0.060 & $0.555(0.300-1.028)$ & 0.061 \\
\hline $\mathrm{APOB}$ & $2.009(1.182-3.414)$ & 0.010 & $1.964(1.151-3.351)$ & 0.013 & $1.978(1.150-3.403)$ & 0.014 \\
\hline $\mathrm{TC} / \mathrm{HDL}$ & $1.232(1.097-1.382)$ & $<0.001$ & $1.210(1.080-1.356)$ & 0.006 & $1.216(1.084-1.364)$ & 0.001 \\
\hline LDL/HDL & $1.376(1.179-1.604)$ & $<0.001$ & $1.358(1.165-1.582)$ & $<0.001$ & $1.363(1.169-1.590)$ & $<0.001$ \\
\hline non-HDL & $1.294(1.160-1.500)$ & 0.001 & $1.279(1.102-1.485)$ & 0.001 & $1.286(1.105-1.496)$ & 0.001 \\
\hline $\mathrm{Al}$ & $1.232(1.097-1.382)$ & $<0.001$ & $1.210(1.097-1.356)$ & 0.001 & $1.216(1.084-1.364)$ & 0.001 \\
\hline $\mathrm{LCl}$ & $1.018(1.010-1.026)$ & $<0.001$ & $1.017(1.008-1.025)$ & $<0.001$ & $1.017(1.008-1.026)$ & $<0.001$ \\
\hline API & $3.685(2.081-6.532)$ & $<0.001$ & $3.290(1.842-5.877)$ & $<0.001$ & $3.619(2.003-6.538)$ & $<0.001$ \\
\hline APOA-1/APOB & $3.159(1.665-5.995)$ & $<0.001$ & $3.076(1.616-5.855)$ & 0.001 & $3.119(1.628-5.979)$ & 0.001 \\
\hline
\end{tabular}

$\mathrm{Cl}$ confidence interval, $\mathrm{OR}$ odds ratio, Model 1:adjustment risk factors including hypertension, diabetes; Model 2: Those variables (hypertension, diabetes, smoking, heart rate, FBG) that were significant $(P<0.05)$ in univariate models were then simultaneously entered into this multivariable model; 
Toft-Petersen et al. [25] conducted a cross-sectional study which found an association between sdLDL concentration and the presence of CAD in the elderly population. They verified that there was relation between AIP and mortality in the elderly. Significantly increased AIP and intima-media thickness of the carotid artery were found in subclinical atherosclerosis in patients on maintenance hemodialysis in Turkey [29].Similar findings of AIP as useful tool for the diagnosis and prognosis of CVD was reported in Morocco population [30]. Although this index has been used to predict the risk of atherosclerosis in hypertensive postmenopausal women in south East Nigeria [17]. However, contrary to our conclusion, Nansseu et al. [18] found AIP may not be an independent factor impacting the risk of CVD through a cross-sectional study conducted among 108 postmenopausal women in Cameroon. At the same time, atherogenic lipoproteins that have been identified, the high apoliprotein B, low apolipoprotein A-1 and the high ratio of apoB: apoA1 also affect the formation and progression of coronary atherosclerosis [31, 32]. In our study, we also find an increased ratio of apoB: apoA1 value was a highly significant risk factor of CAD independent of smoking, hypertension, and diabetes. It remains predictive after adding other confounding factors.In 2002, sdLDL was identified as a major risk factor for CAD by the National Cholesterol Education Program, and the program recommended detection of sdLDL [33]. However, all currently available methods used to detect sdLDL have limitations and suffer from the problem of high cost, which is difficult to popularize in clinical practices like the detection of TG, TC, HDL-C and LDL-C. Nevertheless, the calculation of AIP is easy and AIP effectively reflects the sdLDL level. Therefore, the use of AIP to evaluate the risk of developing atherosclerosis is feasible for the prevention and control of cardiovascular diseases in a community population.

\section{Limitation}

In our study, we found that AIP as useful tool for the diagnosis of CAD in postmenopausal women. However, some interventions such as differences in culture, race, diet, lifestyle, demographic characteristics, laboratory tests and different postmenopausal and metabolic status could also affect AIP values. Especially the role of nutraceuticals on lipid plasma levels is very important. Nutraceuticals are not only effectively able to reduce the burden of the atherosclerosis process, coronary heart disease development, mortality and morbidity caused by CAD, it but also can be able to ameliorate human dyslipidaemia as already demonstrated in the literature by Pietro Scicchitano [34]. A variety of traditional and non-traditional nutraceuticals are used to regulate blood lipids by different molecular mechanisms. Besides, some pharmacological interventions (such as hormone replacement therapy and vitamin D supplementation) could be considered, though most of them have yielded controversial outcomes. Most importantly, oral contraceptives, or medications known to affect lipid metabolism (lipid-lowering drugs, fish oil capsules, Beta-blockers, or diuretics) should be considered. Also the retrospective nature of this paper is a limitation of its design. We made a propensity score matching case-control study to make the data between the case-control group can be compared and try our best to realize the integrity of data and eliminate the incomplete data in the process of data collection, and reflect objective facts as accurately as possible. Dyslipidaemia in our postmenopausal women is indicative of their susceptibility to atherosclerosis and other cardiovascular disorders, therefor, larger, community-based and well designed studies are warranted to better investigate the relationship between AIP and risk of CVD among postmenopausal women.

\section{Conclusion}

The present study suggested that AIP might be a strong marker for predicting the risk of CAD in postmenopausal women.

\section{Abbreviations}

AIP: Atherogenic index of plasma; CAD: Coronary artery disease; CAG: Coronary angiography; DM: Diabetes mellitus; EH: Essential hypertension; LVEF: Left ventricular ejection fraction

\section{Acknowledgements}

We thank the nurse staff of the Department of Cardiology for their help with the blood sampling of the patients.

\section{Funding}

This work was supported by the National Natural Science Foundation of China (81560070).

\section{Availability of data and materials}

Due to confidentiality policies, data will not be shared.

\section{Authors' contributions}

TTW and YG made substantial contributions to study conception and design and to the drafting and critical revision of the manuscript for important intellectual content.

YYZ and YTM made substantial contributions to the study conception and design and critical revision of the manuscript for important intellectual content. XX made substantial contributions to study conception and design, drafting and critical revision of the manuscript for important intellectual content, including study supervision. All authors read and approved the final manuscript.

\section{Ethics approval and consent to participate}

The research protocol was approved by the ethics committee or review committee of the First Affiliated Hospital of Xinjiang Medical University. Because the study was a retrospective cohort study based on real-world situations, there was no need to obtain informed consent from the patients.

\section{Consent for publication}

All authors aggree to publish this work.

\section{Competing interests}

No potential conflicts of interest relevant to this article were reported by any of the authors. None of the funding sources played a role in the design, collection, analysis or interpretation of the data or in the decision to submit the manuscript for publication. The authors declare that they have no competing interests. 


\section{Publisher's Note}

Springer Nature remains neutral with regard to jurisdictional claims in published maps and institutional affiliations.

\section{Author details}

${ }^{1}$ Department of Cardiology, First Clinical Medical College \& First Affiliated Hospital of Xinjiang Medical University, Urumqi 830054, People's Republic of China. ${ }^{2}$ Cadre Ward, First Clinical Medical College \& First Affiliated Hospital of Xinjiang Medical University, Urumqi 830054, People's Republic of China. ${ }^{3}$ Department of Cardiology, First Affiliated Hospital of Zhengzhou University, Zhengzhou 450000, People's Republic of China.

Received: 11 May 2018 Accepted: 17 July 2018

\section{Published online: 22 August 2018}

\section{References}

1. NIH Consensus conference. Triglyceride, high-density lipoprotein, and coronary heart disease. NIH Consensus Development Panel on Triglyceride, High-Density Lipoprotein, and Coronary Heart Disease. JAMA. 1993;269(4):505-10

2. Wilson PW, D'Agostino RB, Levy D, Belanger AM, Silbershatz $H$, Kannel WB. Prediction of coronary heart disease using risk factor categories. Circulation. 1998;97(18):1837-47.

3. Rosenson RS. Low high-density lipoprotein cholesterol and cardiovascular disease: risk reduction with statin therapy. Am Heart J. 2006;151(3):556-63.

4. Aatola $H$, Hutri-Kähönen $N$, Juonala $M$, Laitinen $\Pi$, Pahkala $K$, Mikkilä V, Telama R, Koivistoinen T, Lehtimäki T, Viikari JS, Raitakari OT, Kähönen M. Prospective relationship of change in ideal cardiovascular health status and arterial stiffness: the cardiovascular risk in young Finns study. J Am Heart Assoc. 2014;3(2):e000532

5. Folsom AR, Yatsuya H, Nettleton JA, Lutsey PL, Cushman M. Rosamond WD; ARIC study Investigators.Community prevalence of ideal cardiovascular health, by the American Heart Association definition, and relationship with cardiovascular disease incidence. J Am Coll Cardiol. 2011;57(16):1690-6.

6. Shen S, Lu Y, Qi H, Li F, Shen Z, Wu L, Yang C, Wang L, Shui K, Wang Y, Qiang D, Yun J, Weng X. Association between ideal cardiovascular health and the atherogenic index of plasma. Medicine (Baltimore). 2016; 95(24):e3866.

7. Niroumand S, Khajedaluee M, Khadem-Rezaiyan M, Abrishami M, Juya M Khodaee G, Dadgarmoghaddam M. Atherogenic index of plasma (AIP):a marker of cardiovascular disease. Med J Islam Repub Iran. 2015:29:240.

8. Dobiásová M, Frohlich J. The plasma parameter log (TG/HDL-C) as an atherogenic index: correlation with lipoprotein particle size and esterification rate in apoB-lipoprotein-depleted plasma (FER(HDL)). Clin Biochem. 2001;34(7):583-8.

9. Dobiasova M. AlP-atherogenic index of plasma as a significant predictor of cardiovascular risk: from research to practice. Vnitr Lek. 2006:52(1):64-71.

10. Pahwa MB, Seth S, Seth RK. Lipid profile in various phases of menstrual cycle and its relationship with percentage plasma volume changes. Clin Chim Acta. 1998;273(2):201-7.

11. Warren MP, Halpert S. Hormone replacement therapy: controversies, pros and cons. Best Pract Res Clin Endocrinol Metab. 2004;18(3):317-32.

12. Augoulea A, Mastorakos G, Lambrinoudaki I, Christodoulakos G, Creatsas G Role of postmenopausal hormone replacement therapy on body fat gain and leptin levels. Gynecol Endocrinol. 2005;20(4):227-35.

13. Reckelhoff JF. Basic research into the mechanisms responsible for postmenopausal hypertension. Int J Clin Pract Suppl. 2004;139:13-9.

14. Wu SI, Chou P, Tsai ST. The impact of years since menopause on the development of imAlPred glucose tolerance. J Clin Epidemiol. 2001; 54(2):117-20.

15. Nomenclature and criteria for diagnosis of ischemic heart disease. Report of the Joint International Society and Federation of Cardiology/World Health Organization task force on standardization of clinical nomenclature. Circulation. 1979;59(3):607-9.

16. Gensini GG. A more meaningful scoring system for determining the severity of coronary heart disease. Am J Cardiol. 1983;51(3):606.

17. Nwagha UI, Ikekpeazu EJ, Ejezie FE, Neboh EE, Maduka IC. Atherogenic index of plasma as useful predictor of cardiovascular risk among postmenopausalwomen in Enugu. Nigeria Afr Health Sci. 2010;10(3):248-52.
18. Nansseu JR, Moor VJ, Nouaga ME, Zing-Awona B, Tchanana G, Ketcha A Atherogenic index of plasma and risk of cardiovascular disease among Cameroonianpostmenopausal women. Lipids Health Dis. 2016;15:49.

19. Onat A, Can G, Kaya H, Hergenç G. "Atherogenic index of plasma" (log10 triglyceride/high-density lipoprotein-cholesterol) predicts high blood pressure, diabetes, and vascular events. J Clin Lipidol. 2010;4(2):89-98.

20. Zhu XW, Deng FY, Lei SF. Meta-analysis of Atherogenic index of plasma and other lipid parameters in relation to risk of type 2 diabetes mellitus. Prim Care Diabetes. 2015;9(1):60-7.

21. Lippi G, Montagnana M, Luca Salvagno G, Targher G, Cesare GG. Epidemiological association between uric acid concentration in plasma, lipoprotein(a), and the traditional lipid profile. Clin Cardiol. 2010;33(2):E76-80.

22. Cure MC, Tufekci A, Cure E, Kirbas S, Ogullar S, Kirbas A, Unal H, Yuce S, Cakmak S. Low-density lipoprotein subfraction, carotid artery intima-media thickness, nitric oxide, andtumor necrosis factor alpha are associated with newly diagnosed ischemic stroke. Ann Indian Acad Neurol. 2013;16(4):498-503.

23. Gaál K, Tarr T, Lőrincz H, Borbás V, Seres I, Harangi M, Fülöp P, Paragh G. High-density lipopoprotein antioxidant capacity, subpopulation distribution and paraoxonase-1 activity in patients with systemic lupus erythematosus. Lipids Health Dis. 2016;15:60.

24. Bendzala M, Sabaka P, Caprnda M, Komornikova A, Bisahova M, Baneszova R, Petrovic D, Prosecky R, Rodrigo L, Kruzliak P, Dukat A. Atherogenic index of plasma is positively associated with the risk of all-cause death in elderly women : a 10-year follow-up. Wien Klin Wochenschr. 2017;129(21-22):793-8.

25. Toft-Petersen AP, Tilsted HH, Aarøe J, Rasmussen K, Christensen T, Griffin BA, Aardestrup IV, Andreasen A, Schmidt EB. Small dense LDL particles--a predictor of coronary artery disease evaluated by invasive and CT-based techniques: a case-control study. Lipids Health Dis. 2011;10:21.

26. Grammer TB, Kleber ME, März W, Silbernagel G, Siekmeier R, Wieland H, Pilz S, Tomaschitz A, Koenig W, Scharnagl H. Low-density lipoprotein particle diameter and mortality:The Ludwigshafen risk and cardiovascular health study. Eur Heart J. 2015;36(1):31-8.

27. El Harchaoui K, van der Steeg WA, Stroes ES, Kuivenhoven JA, Otvos JD, Wareham NJ, Hutten BA, Kastelein JJ, Khaw KT, Boekholdt SM. Value of lowdensity lipoprotein particle number and size as predictors of coronary artery disease in apparently healthy men and women: the EPICN or folk prospective population study. J Am Coll Cardiol. 2007:49(5):547-53.

28. Dobiásová M, Frohlich J, Sedová M, Cheung MC, Brown BG. Cholesterol esterification and atherogenic index of plasma correlate with lipoprotein size and findings on coronary angiography. J Lipid Res. 2011;52(3):566-71.

29. Yildiz G, Duman A, Aydin H, Yilmaz A, Hür E, Mağden K, Cetin G, Candan F. Evaluation of association between atherogenic index of plasma and intimamedia thickness of the carotid artery for subclinic atherosclerosis in patients on maintenance hemodialysis. Hemodial Int. 2013;17(3):397-405.

30. Essiarab F, Taki H, Lebrazi H, Sabri M, Saïle R. Usefulness of lipid ratios and atherogenic index of plasma in obese Moroccan women with or without metabolic syndrome. Ethn Dis. 2014;24(2):207-12.

31. Crouse JR, Parks JS, Schey HM, Kahl FR. Studies of low density lipoprotein molecular weight in human beings with coronary artery disease. J Lipid Res. 1985;26(5):566-74.

32. Biswas S, Ghoshal PK, Mandal SC, Mandal N. Association of low-density lipoprotein particle size and ratio of different lipoproteins andapolipoproteins with coronary heart disease. J Cardiol. 2008;52(2):118-26.

33. SoRelle R. ATP III calls for more intensive low-density lipoprotein lowering in target groups. Circulation. 2002;106(25):e9068-8.

34. Scicchitano P, Cameli M, Maiello M, Modesti PA, Muiesan ML, Novo S, Palmiero P, Saba PS, Pedrinelli R, Ciccone MM, on behalf of the "Gruppo di Studio Ipertensione, Prevenzione e Riabilitazione", Societa' Italiana di Cardiologia. Nutraceuticals and dyslipidaemia: Beyond the common therapeutics. J Funct Foods. 2014;6:11-32. 